\title{
Robust Step-up DC/AC conversion with a Full-Bridge Non-Inverting Buck-Boost
}

\author{
Domingo Biel, Enric Fossas and Josep M. Olm
}

\begin{abstract}
The substitution of the original switches by a full-bridge in a Non-Inverting Buck-Boost converter results in an inverter capable of performing step-down and step-up tasks under sliding mode control. Furthermore, semi-infinite programming techniques are used to minimize power loses while preventing control action saturation. The performance of the inverter is shown to be robust in front of load perturbations. The procedure assumes known bounds for the disturbances, as well as full state knowledge. Realistic simulations validate the proposed scheme.
\end{abstract}

\section{INTRODUCTION}

The possibility of using nonlinear DC-DC switching power converters as source inverters has been thoroughly studied during the last twenty years. The main reason for this is that the conventional Full-Bridge Buck converter must incorporate a transformer to adequately perform stepup tasks, thus resulting in a significative increase of the weight and size of the power supply equipment. When using nonlinear converters, efforts are handicapped by the nonminimum phase character shown by these devices when direct control over the output voltage is exerted [1].

First attempts to solve the problem by means of a currentbased indirect control in boost and buck-boost DC-AC inverters [2],[3] have lead to systems with sensitivity to external perturbations and parameter uncertainties. Moreover, direct control strategies of the output voltage that include passivitybased schemes [4] and PID-type sliding mode controllers [5] have been used for regulation purposes. PI controllers also offer interesting performance in full-bridge nonlinear inverters [6],[7],[8],[9]. However, it is well known that PI control designs are based on a small signal model; this leads to output waveforms being sensitive to power stage parameter variations, such as the output load.

The Full-bridge Non-Inverting Buck-Boost inverter, which is essentially achieved by a full-bridge inverter in series with a Boost converter, has two control inputs. Hence, it is possible to design a sliding mode control strategy which is able to yield robust tracking of periodic signals by the output voltage and, at the same time, maintain the input current regulated at a prescribed level. The proposal uses a fullstate reference profile-based switching surface that does not depend on the plant parameters. The inverter is assumed to

D. Biel is partially supported by CICYT grant DPI2006-15627-C03-01. E. Fossas and J.M. Olm are partially supported by MCyT grant DPI200406871-C02-02

The authors are with the Institute of Industrial and Control Engineering, Technical University of Catalonia, Barcelona, Spain bieldeel.upc.edu,

\{enric.fossas, josep.olm\}@upc.edu undergo possible load variations in a set with known bounds Restrictions for candidate signals to be tracked are derived by demanding non-saturation of the control action, which has fixed control gains, in the steady state.

Existing literature dealing with the Non-Inverting BuckBoost converter contains results for regulation tasks [10], [11]. In [12], sliding modes are used to achieve output voltage tracking of sinusoidal signals with offset: the performance is shown to be robust under resistive load variation in a set with known bounds. The theoretical development presented in that article is applied in this paper to the FullBridge Non-Inverting Buck-Boost converter, this leading to a robust step-up/down DC/AC inversion.

It is already known that proper energy transfer constitutes the main goal of power converters, this meaning good efficiency and high output signal quality. Maximizing power efficiency requires minimization of the Root Mean Square (RMS) of the current flowing in the switching converter, this leading to two different effects, namely, optimization of the loses due to the power switching and minimization of the resistive loses in the inductors. Hence, the design incorporates a procedure to reduce power loses based in semiinfinite programming theory [13] and assuming known upper and lower bounds for the load variation; this technique has been successfully applied to a Full-Bridge Boost converter in [14] and also to a Full-Bridge Non-Inverting Buck-Boost in [12]. Finally, the theoretical development is validated by carrying out illustrative simulations with the realistic power electronics software package PSIM.

The article is structured as follows. The mathematical model of the Full-bridge Non-Inverting Buck-Boost is established in Section II. A sliding mode control strategy to achieve the output voltage tracking target is developed in Section III. The selection of a current reference profile that minimizes power loses and guarantees non-saturation of the controller is studied in Section IV. Simulation results are presented in Section V, while Conclusions and some suggestions for further research are in Section VI.

\section{THE FULL-BRIDGE NON-INVERTING BUCK-BOOST CONVERTER}

The Non-Inverting Buck-Boost converter, depicted in Figure 1 , can be modelled as a two-dimensional, bilinear system with the inductor current $i_{\mathrm{L}}$ and the capacitor voltage $v_{\mathrm{C}}$ as 


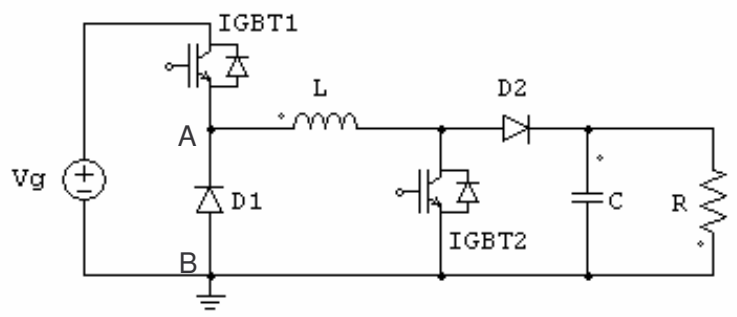

Fig. 1. Non-Inverting Buck-Boost converter.

state variables:

$$
\begin{aligned}
L \frac{d i_{\mathrm{L}}}{d \tau} & =V_{A B}-(1-q) v_{\mathrm{C}} \\
C \frac{d v_{\mathrm{C}}}{d \tau} & =-\frac{v_{\mathrm{C}}}{R}+(1-q) i_{\mathrm{L}} .
\end{aligned}
$$

The control actions envisage the possibility of forcing $V_{A B}=$ $V_{g}$ or $V_{A B}=0$ at will, and $q=0, q=1$ as well. Introducing the control gains $u_{1}$ and $u_{2}=1-q$, both of them taking values in the discrete set $\{0,1\}$, system (1),(2) results in

$$
\begin{aligned}
L \frac{d i_{\mathrm{L}}}{d \tau} & =V_{g} u_{1}-v_{\mathrm{C}} u_{2} \\
C \frac{d v_{\mathrm{C}}}{d \tau} & =-\frac{v_{\mathrm{C}}}{R}+i_{\mathrm{L}} u_{2} .
\end{aligned}
$$

For a systematic analysis it is advisable to minimize the number of parameters of the system. This purpose may be achieved with the change of variables:

$$
x_{1}=\frac{1}{V_{\mathrm{g}}} \sqrt{\frac{L}{C}} i_{\mathrm{L}}, \quad x_{2}=\frac{1}{V_{\mathrm{g}}} v_{\mathrm{C}}, \quad t=\frac{1}{\sqrt{L C}} \tau,
$$

and the introduction of

$$
\lambda=\frac{1}{R} \sqrt{\frac{L}{C}}>0,
$$

which make the system dimensionless:

$$
\begin{aligned}
& \dot{x}_{1}=u_{1}-x_{2} u_{2} \\
& \dot{x}_{2}=-\lambda x_{2}+x_{1} u_{2} .
\end{aligned}
$$

The converter is assumed to undergo possible load variations in the bounded set $\left[R_{\min }, R_{\max }\right]$, this yielding $\lambda \in \Lambda=$ $\left[\lambda_{\min }, \lambda_{\max }\right]$.

A detailed study of the dynamics of the Non-Inverting Buck-Boost converter reveals the impossibility of performing inversion tasks [12]. This situation may be overcome with the replacement of each of the original switches by a full bridge of switches that allows bi-directional current flows. The resulting converter is shown in Figure 2. However, the new system dynamics are still modelled by (5),(6), the change being in the fact that now the control gains $u_{1}, u_{2}$ take values in the set $\{-1,1\}$.

Assume that the control goal is the tracking of certain $T$-periodic reference signals $x_{1 d}(t), x_{2 d}(t)$ by the state

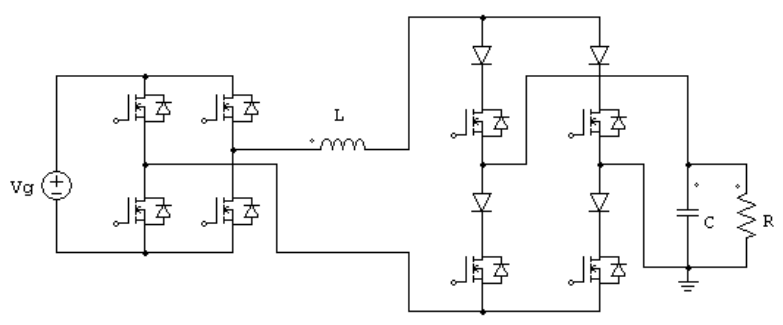

Fig. 2. Full-bridge Non-Inverting Buck-Boost converter.

variables $x_{1}, x_{2}$, respectively. Hence, in the steady state it must be

$$
\begin{aligned}
& \dot{x}_{1 d}=u_{1 N}-x_{2 d} u_{2 N} \\
& \dot{x}_{2 d}=-\lambda x_{2 d}+x_{1 d} u_{2 N},
\end{aligned}
$$

where $u_{1 N}, u_{2 N}$ are the average tracking controls that yield $x_{1}=x_{1 d}(t), x_{2}=x_{2 d}(t)$.

Remark 1: Note that the control saturation avoidance condition, i.e. $u_{1 N}, u_{2 N} \in[-1,1]$, entails restrictions on admissible target functions: on the one hand it must be $x_{1 d}(t) \neq 0, \forall t \geq 0$, and, on the other hand, $\forall t \in[0, T]$ and $\forall \lambda \in \Lambda$,

$$
\begin{array}{r}
-1<u_{1 N}=\frac{x_{1 d} \dot{x}_{1 d}+x_{2 d}\left(\dot{x}_{2 d}+\lambda x_{2 d}\right)}{x_{1 d}}<1 \\
-1<u_{2 N}=\frac{\dot{x}_{2 d}+\lambda x_{2 d}}{x_{1 d}}<1 .
\end{array}
$$

The fulfillment of (9),(10) guarantees unsaturation of the control action in the steady state.

Finally, using error variables $e_{i}=x_{i}-x_{i d}(t)$, and $e_{u_{i}}=$ $u_{i}-u_{i N}, i=1,2$, equations (5),(6) result in

$$
\begin{aligned}
& \dot{e}_{1}=e_{u_{1}}-x_{2 d} e_{u_{2}}-e_{2} u_{2} \\
& \dot{e}_{2}=-\lambda e_{2}+x_{1 d} e_{u_{2}}+e_{1} u_{2} .
\end{aligned}
$$

\section{SLIDING CONTROL OF THE FULL-BRIDGE NON-INVERTING BUCK-BOOST}

First of all, consider that the following assumption is fulfilled from now on:

\section{Assumption A.}

(i) The state vector reference profiles $x_{1 d}(t), x_{2 d}(t)$, are $\mathcal{C}^{2}$ and $T$-periodic.

(ii) $x_{1 d}(t) \neq 0, \forall t \geq 0$.

(iii) The nominal tracking controls $u_{1 N}, u_{2 N}$, defined in (9), (10), respectively, lie inside the $\mathbb{R}^{2}$ region $[-1,1] \times$ $[-1,1], \forall(t, \lambda) \in[0, T] \times \Lambda$.

Let now $\sigma:=\sigma(e, t) \in \mathbb{R}^{2}$, with

$$
\begin{aligned}
& \sigma_{1}=-e_{1} \\
& \sigma_{2}=x_{2 d}(t) e_{1}-x_{1 d}(t) e_{2},
\end{aligned}
$$

be a switching surface for system (11),(12). 
By Assumption A.(ii), $x_{1 d}(t) \neq 0$. Hence,

$$
\sigma=\left(\begin{array}{cc}
-1 & 0 \\
x_{2 d} & -x_{1 d}
\end{array}\right)\left(\begin{array}{l}
e_{1} \\
e_{2}
\end{array}\right)=0 \Longleftrightarrow\left\{\begin{array}{l}
e_{1}=0 \\
e_{2}=0
\end{array}\right.
$$

additionally, it is straightforwardly verifiable that the equivalent controls coincide with the nominal controls given in (9),(10), i.e.

$$
u_{1 e q}=u_{1 N}, \quad u_{2 e q}=u_{2 N} .
$$

The switching logic that provides the tracking target is given in next Proposition:

Proposition 1: Let Assumption A hold. Let also $\sigma$ defined in (13) be a switching surface for the system modelled by equations (11),(12). Then, the control law

$u_{1}=\left\{\begin{array}{rl}1 & \text { if } \sigma_{1}>0 \\ -1 & \text { if } \sigma_{1}<0\end{array}, \quad u_{2}=\left\{\begin{array}{rl}1 & \text { if } \sigma_{2}>0 \\ -1 & \text { if } \quad \sigma_{2}<0\end{array}\right.\right.$,

yields sliding modes on $\sigma(e, t)=0$ and the ideal sliding dynamics results in $x_{1}=x_{1 d}(t), x_{2}=x_{2 d}(t)$.

Proof: Let $W(t)$ be the time dependent, real, positive definite, symmetric matrix

$$
W(t)=\frac{1}{x_{1 d}^{2}(t)}\left(\begin{array}{cc}
x_{1 d}^{2}(t)+x_{2 d}^{2}(t) & x_{2 d}(t) \\
x_{2 d}(t) & 1
\end{array}\right) .
$$

Then,

$$
V(\sigma, t)=\frac{1}{2} \sigma^{\top} W(t) \sigma
$$

is a smooth, positive definite, quadratic function. Furthermore, as the eigenvalues of $W(t)$ are positive real functions of $t$, it results that (see [15], for example),

$$
0 \leq \kappa_{\min }(t)\|\sigma\|^{2} \leq 2 V(\sigma, t) \leq \kappa_{\max }(t)\|\sigma\|^{2}
$$

where $\kappa_{\min }(t), \kappa_{\max }(t)$ are the smallest and largest eigenvalues of $W(t)$, respectively. The continuity and $T$-periodicity of such eigenvalues allow us to conclude that they achieve a maximum and a minimum value in $[0, T]$, i.e. there exist real, positive constants $\rho_{m}, \rho_{M}$ fulfilling

$$
\begin{aligned}
& 2 \rho_{m} \leq \min _{t \in[0, T]}\left\{\kappa_{\text {min }}(t)\right\} \\
& 2 \rho_{M} \geq \max _{t \in[0, T]}\left\{\kappa_{\text {max }}(t)\right\} .
\end{aligned}
$$

Therefore, $V(\sigma, t)$ is lower and upper bounded in each sphere $\|\sigma\|=R$ inside a neighborhood of $\sigma=0$ by positive quantities depending only on $R$, and these lower and upper bounds $h_{R}=\rho_{m} R^{2}, H_{R}=\rho_{M} R^{2}$, respectively, are such that

$$
\lim _{R \rightarrow 0} H_{R}=0, \quad \lim _{R \rightarrow \infty} h_{R}=\infty .
$$

In order to evaluate the derivative of $V(\sigma, t)$ along the trajectories of (11),(12), note that

$$
V(\sigma, t)=\frac{1}{2} e^{\top} e
$$

Thus,

$$
\begin{aligned}
\dot{V} & =e_{1}\left[e_{u_{1}}-x_{2 d} e_{u_{2}}-e_{2} u_{2}\right]+ \\
& +e_{2}\left[-\lambda e_{2}+x_{1 d} e_{u_{2}}+e_{1} u_{2}\right]= \\
& =-\lambda e_{2}^{2}+e_{1} e_{u_{1}}+\left(x_{1 d} e_{2}-x_{2 d} e_{1}\right) e_{u_{2}} \leq \\
& \leq-\sigma_{1}\left(u_{1}-u_{1 e q}\right)-\sigma_{2}\left(u_{2}-u_{2 e q}\right) .
\end{aligned}
$$

Note that, by Assumption $\mathrm{A}, u_{1 e q}$ and $u_{2 e q}$ are continuous, $T$-periodic and both of them lay inside $(-1,1)$; therefore, they reach maximum and minimum values $u_{i e q}^{+}$and $u_{i e q}^{-}$, $i=1,2$, therein, respectively. Let $\epsilon_{1}, \epsilon_{2}$ be

$$
\epsilon_{i}=\inf \left\{1-u_{i e q}^{+},\left|-1-u_{i e q}^{-}\right|\right\}, \quad i=1,2 .
$$

Then, the proposed switching logic yields

$$
\dot{V}(\sigma, t) \leq-\epsilon_{1}\left|\sigma_{1}\right|-\epsilon_{2}\left|\sigma_{2}\right| \leq-\alpha\left(\left|\sigma_{1}\right|+\left|\sigma_{2}\right|\right),
$$

with $\alpha=\inf \left\{\epsilon_{1}, \epsilon_{2}\right\}$. By norm equivalence in $\mathbb{R}^{n}$, there exists $\hat{\alpha}>0$ such that

$$
\dot{V}(\sigma, t) \leq-\hat{\alpha} \sqrt{\sigma_{1}^{2}+\sigma_{2}^{2}}=-\hat{\alpha}\|\sigma\|
$$

Then, a stable sliding mode along the intersection of the discontinuity surfaces $\left\{\sigma_{1}=0\right\} \cap\left\{\sigma_{2}=0\right\}$ occurs [16].

Remark 2: Notice from (11), (12) that, if $x_{1 d} \neq 0$, every perturbation of the system satisfies the matching condition [17]. Hence, the induced sliding regimes satisfy a so-called strong invariance property [17], which results in the ideal sliding dynamics being independent of the perturbation signal, thus guaranteeing robustness. However, the disturbance may affect the fulfillment of (9),(10): therefore, Assumption A.(iii) has to be preserved in order to prevent the loss of sliding motion on $\sigma(e, t)=0$.

\section{POWER LOSS MINIMIZATION}

Due to both technical and economical reasons, it is extremely convenient to reduce as much as possible power loses in the converters. In this sense, in [12] the authors have developed a procedure to reduce power loses in a class of switched converters through the minimization of the RMS current reference profile. The technique is applied below to the Full-Bridge Non-Inverting Buck-Boost converter.

The power dissipated in a resistive circuit element is directly proportional to the square of the Root Mean Square (RMS) of the current that flows through the element. Moreover, as the target is the tracking of $T$-periodic output voltage references $x_{2 d}(t)$, it is reasonable to search for inductor current reference profiles $x_{1 d}(t)$ which are also $T$-periodic. Hence, assume a truncated Fourier series development for $x_{1 d}$ :

$$
x_{1 d}(t)=a_{0}+\sum_{k=1}^{r} a_{k} \cos k \omega t+b_{k} \sin k \omega t,
$$

with $\omega=2 \pi T^{-1}$. We may minimize its RMS

$$
\begin{aligned}
F\left(a_{0}, a_{1}, \ldots, b_{r}\right) & =\sqrt{\frac{1}{T} \int_{0}^{T} x_{1 d}^{2}(t) d t}= \\
& =\sqrt{a_{0}^{2}+\sum_{k=1}^{k=r} \frac{a_{k}^{2}+b_{k}^{2}}{2}},
\end{aligned}
$$

subjected to the restrictions imposed by (9) and (10). Then, the problem to be solved consists of choosing $a_{0}, a_{1}, b_{1}, \ldots$, $a_{r}, b_{r}$ so that function $F$ defined in (15) reaches a minimum on the domain defined by inequalities (9) and (10). 
The minimization procedure is based on the following result:

Theorem 1: Let the scalar functions $\phi_{i}: \mathbb{R}^{p} \times \mathbb{R}^{q} \longrightarrow \mathbb{R}$, $i=1, \ldots, m$, be continuously differentiable, and let the set

$$
Z^{P}=\left\{z \in \mathbb{R}^{p}, \quad \phi_{i}(z, y) \leq 0, \quad i=1, \ldots, m\right\}
$$

be nonempty. Then, there exists $z \in \mathbb{R}^{p}$ that minimizes the real valued function

$$
F(z)=\|z\|
$$

on the domain defined by $Z^{P}$.

Proof: See [12].

In order to simplify notation, let us denote

$$
x_{1 d}(z, t)=z_{1}+\sqrt{2} \sum_{k=1}^{r} z_{2 k} \cos k \omega t+z_{2 k+1} \sin k \omega t,
$$

with $z \in \mathbb{R}^{2 r+1}, t \in \mathbb{R}$, which has an RMS given by

$$
F(z)=\|z\| \text {. }
$$

Moreover, set $y=(t, \lambda) \in \mathbb{R}^{2}$ and let us define

$$
\begin{aligned}
& f(y)=f(t, \lambda)=\dot{x}_{2 d}(t)+\lambda x_{2 d}(t) \\
& g(y)=g(t, \lambda)=x_{2 d}(t)\left[\dot{x}_{2 d}(t)+\lambda x_{2 d}(t)\right] .
\end{aligned}
$$

Straightforward calculation allows one to prove the following result:

Proposition 2: Let $Y=[0, T] \times \Lambda$ be a compact subset of $\mathbb{R}^{2}$, and consider the scalar functions

$$
\begin{aligned}
\phi_{1}(z, y)= & \phi_{1}(z, t, \lambda)=g(t, \lambda)+ \\
& +x_{1 d}(z, t)\left[\frac{\partial x_{1 d}(z, t)}{\partial t}-1\right] \\
\phi_{2}(z, y)= & \phi_{2}(z, t, \lambda)=-g(t, \lambda)+ \\
& -x_{1 d}(z, t)\left[\frac{\partial x_{1 d}(z, t)}{\partial t}+1\right] \\
\phi_{3}(z, y)= & \phi_{3}(z, t, \lambda)=-\left[x_{1 d}(z, t)-f(t, \lambda)\right] \\
\phi_{4}(z, y)= & \phi_{4}(z, t, \lambda)=-\left[x_{1 d}(z, t)+f(t, \lambda)\right],
\end{aligned}
$$

where $x_{1 d}, f(t, \lambda)$ and $g(t, \lambda)$ are defined in (17) and (18), respectively. If $\phi_{i}(z, y)<0, \forall y \in Y, i=1,2,3,4$, then Assumption A.(iii) holds.

Proposition 3: Let Assumption A hold, and let also $Y=$ $[0, T] \times \Lambda$ be a compact subset of $\mathbb{R}^{2}$. Then,

$$
F(z)=\|z\|
$$

reaches a minimum on the domain

$Z^{P}=\left\{z \in \mathbb{R}^{2 r+1}, \phi_{i}(z, y) \leq 0, \forall y \in Y, \forall i=1,2,3,4\right\}$.

Proof: The continuous differentiability of $\phi_{i}(z, y)$, $i=1,2,3,4$, follows immediately from its own definition and from Assumption A. Furthermore, $Y$ being compact, the continuous, real valued functions $|f(y)|$ and $|g(y)|$ reach maximum values in $Y$, i.e. there exist $f_{M}, g_{M} \geq 0$ such that:

$$
f_{M}=\max _{y \in Y}\{|f(y)|\}, \quad g_{M}=\max _{y \in Y}\{|g(y)|\} .
$$

Setting $z_{2}=\cdots=z_{2 r+1}=0$ in (19),(20),(21),(22) one gets:

$$
\begin{aligned}
& \phi_{1}\left(z_{1}, 0, \ldots, 0 ; y\right)=g(y)-z_{1}, \\
& \phi_{2}\left(z_{1}, 0, \ldots, 0 ; y\right)=-g(y)-z_{1}, \\
& \phi_{3}\left(z_{1}, 0, \ldots, 0 ; y\right)=f(y)-z_{1}, \\
& \phi_{4}\left(z_{1}, 0, \ldots, 0 ; y\right)=-f(y)-z_{1} .
\end{aligned}
$$

Then, the $\mathbb{R}^{2 r+1}$ subset

$$
\hat{Z}_{1}^{P}=\left\{\left(z_{1}, 0, \ldots, 0\right) \in \mathbb{R}^{2 r+1} ; z_{1} \geq \max \left\{f_{M}, g_{M}\right\}\right\}
$$

is, trivially, a non-empty subset of $Z^{P}$. Finally, the result follows from direct application of Theorem 1.

\section{SIMULATION RESULTS}

The power electronics software PSIM is used to carry out the simulations. The parameters of the Full-Bridge NonInverting Buck-Boost converter are: a DC voltage source of $V_{\mathrm{g}}=50 \mathrm{~V}$, a nominal output resistance of $R=5 \Omega$, an inductance of $L=1 \mathrm{mH}$ with an internal resistance of $0.01 \Omega$ and a capacitor of $C=60 \mu \mathrm{F}$ with an internal resistance of $0.01 \Omega$. Each switch is implemented by means of an IGBT with a saturation voltage of $2 \mathrm{~V}$ and a power diode with a voltage drop of $0.5 \mathrm{~V}$.

The sliding mode controllers require the use of multipliers, sums and comparators which can be implemented by means of analog techniques. A detailed description of the procedure may be found in [18]. In order to provide variable switching frequencies in the standard range, the sliding mode controllers use hysteresis cycle comparators [19], [20], with hysteresis cycles of $0.02 \mathrm{~V}$ for $\sigma_{1}$ and $0.4 \mathrm{~V}$ for $\sigma_{2}$, as well as zero order holders. Hence, the maximum switching frequency is limited to $120 \mathrm{KHz}$. Expressions of $\sigma_{1}$ and $\sigma_{2}$ may be found in (13).

The output voltage reference for tracking is

$$
v_{\mathrm{Cd}}(\tau)=100 \sin 2 \pi \nu \tau,
$$

with $\nu=50 H z$. The values of the corresponding normalized variables are:

$$
x_{2 d}(t)=2 \sin \omega t,
$$

with $\omega=0.0770$, corresponding to a normalized period of $T=81.65$. The converter is assumed to undergo load variations up to $100 \%$ of the nominal value, which results in $\lambda$ varying in the set $\left[\lambda_{\min }, \lambda_{\max }\right]=[0.4082,0.8165]$. The input current profile is chosen to be

$$
x_{1 d}(t)=a_{0}+a_{1} \cos \omega t+b_{1} \sin \omega t+a_{2} \cos 2 \omega t+b_{1} \sin 2 \omega t .
$$

The optimization problem is solved by means of the function $f$ seminf, available in the Optimization Toolbox of MATLAB. Essentially, the routine uses cubic and quadratic interpolation techniques to estimate peak values in the semiinfinite constraints. The peak values are used to form a set of constraints that are supplied to a sequential quadratic programming (SQP) method.

Table I contains the optimum values for the reference parameters provided by MATLAB, as well as the corresponding RMS values, in normalized variables, for constant and periodic references. The latter is defined by Fourier series 
truncated at the second harmonic. Note that the use of a periodic reference for the inductor current yields a RMS reduction of $34.60 \%$ with respect to a constant reference. In terms of power consumption, this amount grows to $57.23 \%$.

TABLE I

OPTIMIZED PARAMETERS FOR THE INDUCTOR CURRENT REFERENCE AND ASSOCIATED RMS IN NORMALIZED VARIABLES

\begin{tabular}{||c||c|c|c|c|c|c||}
\hline$x_{1 d}(t)$ & $a_{0}$ & $a_{1}$ & $b_{1}$ & $a_{2}$ & $b_{2}$ & RMS \\
\hline \hline Constant & 3.2731 & 0 & 0 & 0 & 0 & 3.2731 \\
\hline Periodic & 1.9416 & 0 & 0 & -1.1725 & 0.5 & 2.1406 \\
\hline
\end{tabular}

When carrying out realistic simulations of the converter performance, the DC terms of the inductor current references depicted in Table I have to be slightly increased for sliding motion to be induced. This is a foreseeable effect of unmodelled dynamics and parasitic resistances. Table II provides the ideal and actual values of the DC components of the inductor current references, as well as the RSM values, in Ampères. The percentage reduction of RSM and power loss are similar to the above reported for the ideal case. Furthermore, for the particular case we are dealing the coefficients $a_{1}$ and $b_{1}$ of the first harmonic of the current reference profile are very low. Due to this fact, the simulations have been carried out setting $a_{1}=b_{1}=0$.

TABLE II

IDEAL AND REAL VALUES FOR THE DC TERM OF THE INDUCTOR CURRENT REFERENCE AND ASSOCIATED RMS

\begin{tabular}{||c||c|c|c|c||}
\hline$i_{\text {Ld }}(\tau)$ & $i_{\text {Ld0 }}$ (id.) & $i_{\text {Ld0 }}($ real) & RMS (id.) & RMS (real) \\
\hline \hline Constant & 40.09 & 64.00 & 40.09 & 64.00 \\
\hline Periodic & 23.78 & 44.00 & 26.22 & 45.30 \\
\hline
\end{tabular}

Figure 3 depicts the output voltage tracking the command profile for a constant inductor current reference, which is also captured. Furthermore, the plot includes the load current: its jumps indicate the presence of a resistive load disturbance. Figure 4 is analogous to Figure 3, except in the fact that now a variable inductor current reference is being used. The THD values for constant and variable inductor current references are both 0.02 .

Typical applications of DC/AC converters deal not only with resistive loads but also with nonlinear loads. Figure 5 contains a simulation of output voltage tracking with a variable inductor current reference in the presence of a nonlinear load consisting of a Full-Wave Rectifier with a capacitor of $8 \mathrm{mF}$ and a resistive load of $24 \Omega$. The THD is 0.025 .

\section{CONCLUSIONS AND SUGGESTIONS FOR FURTHER RESEARCH}

This article presents an inverter obtained from the replacement of the original switches by a full-bridge in a Non-Inverting Buck-Boost converter. Operating under sliding mode control, the converter performance involves robust step-down as well as step-up tasks in presence of resistive loads under the assumption of known bounds for the load

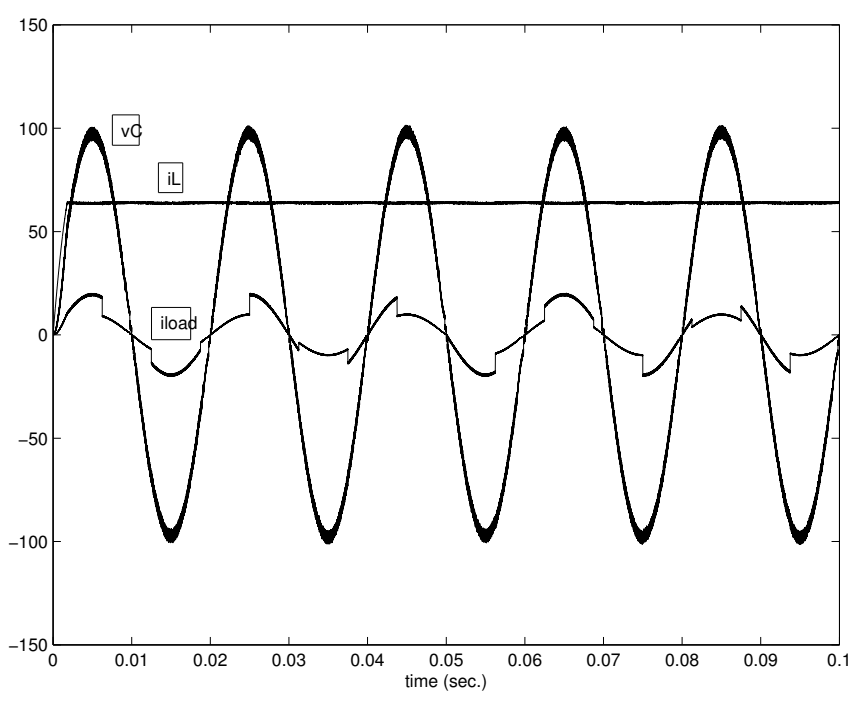

Fig. 3. Output voltage tracking with constant inductor current reference

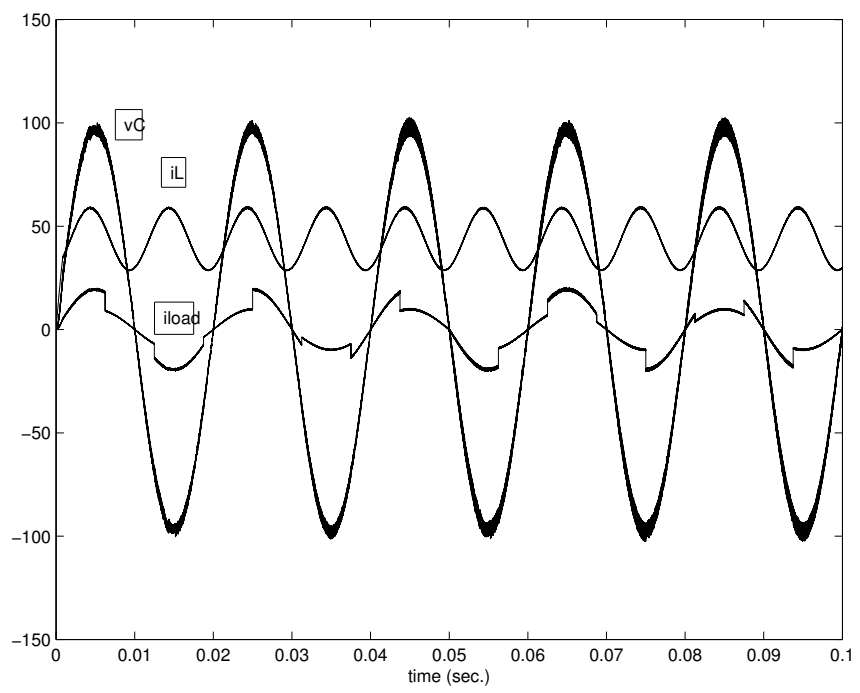

Fig. 4. Output voltage tracking with variable inductor current reference

variation. Using semi-infinite programming techniques, the input current reference is adequately chosen in order to minimize power loses and, at the same time, prevent control saturation. Realistic simulations carried out with PSIM validate the proposal.

Further research may address the performance of the inverter in front of nonlinear loads, for which promising preliminary simulation results have been obtained.

\section{REFERENCES}

[1] E. Fossas and J.M. Olm, Asymptotic Tracking in DC-to-DC Nonlinear Power Converters, Discrete and Continuous Dynamical Systems Series B, vol. 2, 2002, pp. 295-307.

[2] R.O. Cáceres and I. Barbi, A boost DC-AC converter: analysis, design and experimentation, IEEE Trans. Power Electronics, vol. 14, 1999, pp. 134-141.

[3] C. Hernández, N. Vázquez, J. Álvarez and J. Arau, "Modified passivebased control law for the boost inverter", in Proc. of the IEEE 


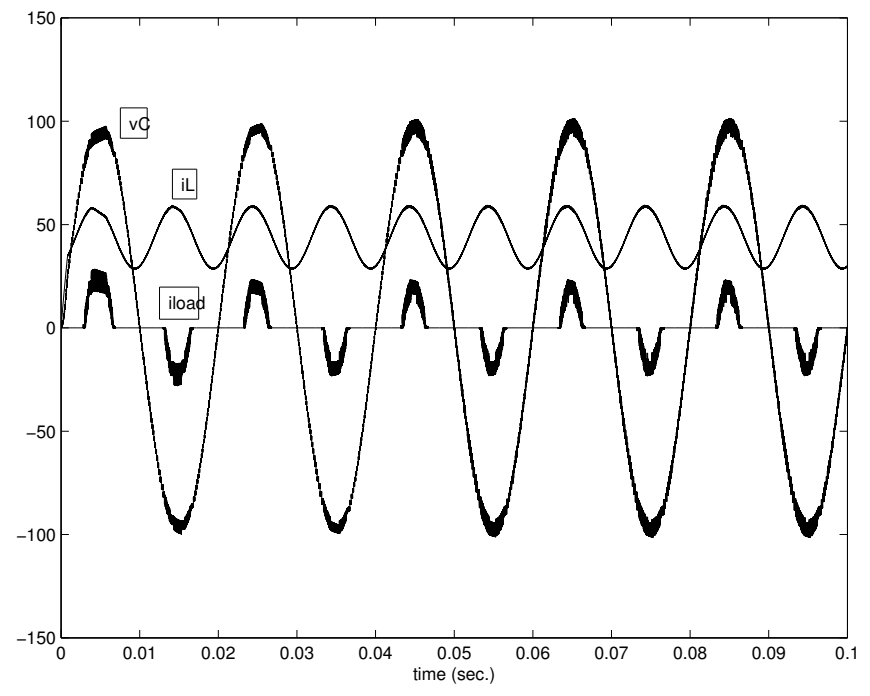

Fig. 5. Output voltage tracking with variable inductor current reference and nonlinear load

International Symposium on Industrial Electronics, vol. 2, 2003, pp. 764-768.

[4] H. Rodríguez, R. Ortega, G. Escobar and N. Baranov, A robustly stable output feedback saturated controller for the boost DC-to-DC converter, Systems \& Control Letters, vol. 40, 2000, pp. 1-8.

[5] S.C. Tan, Y.M. Lai and C.K. Tse, Implementation of Pulse-WidthModulation Based Sliding Mode Controllers for Boost Converters, IEEE Power Electronics Letters, vol. 3, 2005, pp. 130-135.

[6] T.J. Liang, J.L. Shyu and J.F. Chen, "A novel DC-AC boost inverter", in 37th Intersociety Energy Conversion Engineering Conference, 2002, pp. 629-634.
[7] N. Vázquez, D. Cortés, C. Hernández, J. Álvarez, J. Arau and Jq. Álvarez, "A new non-linear control strategy for the boost inverter", in Proc. of the 34th Annual IEEE Power Electronics Specialist Conference, vol. 3, 2003, pp. 1403-1407.

[8] P. Sanchis, A. Ursúa, E. Gubía and L. Marroyo, Design and experimental operation of a control strategy for the buckboost DCAC inverter, IEE Proc. Electr. Power Appl., vol. 152, 2005, pp. 660-668.

[9] P. Sanchis, A. Ursúa, E. Gubía and L. Marroyo, Boost DCAC inverter: a new control strategy, IEEE Transactions on Power Electronics, vol. 20, 2005, pp. 343-353.

[10] B. Sahu and G.A. Rincón-Mora, A low voltage, dynamic, noninverting, synchronous buck-boost converter for portable applications, IEEE Trans. on Power Electronics, vol. 19, 2004, pp. 443-452.

[11] M. Gaboriault and A. Notman, "A high efficiency, noninverting, buckboost DC-DC converter", in Proc. of the 9th Annual IEEE Applied Electronics Conference and Exposition, 2004, pp. 1411-1415.

[12] J.M. Olm, D. Biel, E. Fossas, A. Zinober and L.M. Sanz, Sliding motion, robust control and power loss minimization in a class of nonlinear switched converters, to appear in Int. J. Control, 2007.

[13] R. Hettich and K.O. Kortanek, Semi-infinite programming: theory, methods and applications, SIAM Review, vol. 35, 1993, pp. 380-429.

[14] L.M. Sanz, A.S.I. Zinober, D. Biel, E. Fossas and J.M. Olm, "Sliding Control and Optimization in a Full Bridge Boost Converter", in Taming Heterogeneity and Complexity of Embedded Control: CTS-HYCON Workshop on Nonlinear and Hybrid Control, pp. 673-688, ISTE 2007.

[15] C.T. Chen, Linear System Theory and Design, Holt, Rinehart and Winston, NY, 1984.

[16] V.I. Utkin, Sliding Modes in Control Optimization, Springer-Verlag, Berlin, Heidelberg, 1992.

[17] H. Sira-Ramírez, Differential Geometric Methods in Variable Structure Control, Int. J. Control, vol. 48, 1998, pp. 1359-1390.

[18] D. Biel and E. Fossas, "SMC applications in power electronics", in Variable structure systems: from principles to implementation, pp. 265 293, The Institution of Electrical Engineers, London, 2004.

[19] F. Bilalović, O. Mušić and A. S̆abanović, "Buck Converter Regulator Operating in Sliding Mode", in Proc. of the PCI, 1983, pp. 331-340.

[20] H. Bühler, Réglage par mode de glissement, Presses Polytechniques Romandes, Lausanne, 1986. 